\title{
KRAS mutation in secondary malignant histiocytosis arising from low grade follicular lymphoma
}

\author{
Sarah M. Choi ${ }^{1,2}$, Aleodor A. Andea', Min Wang ${ }^{2}$, Amir Behdad', Lina Shao², Yanming Zhang ${ }^{3}$, Xinyan Lu', \\ David Dittmann ${ }^{4}$, Juan Castro ${ }^{4}$, Yi-Hua Chen ${ }^{1}$ and Juehua Gao ${ }^{1 *}$ (D)
}

\begin{abstract}
Background: Transformation of follicular lymphoma most typically occurs as diffuse large B-cell lymphoma, however other forms of transformation such as classic Hodgkin lymphoma and lymphoblastic transformation can occur. Secondary malignant histiocytosis also represents a rare form of transformation, which is thought to occur due to a process of transdifferentiation whereby the lymphoma cells exhibit lineage plasticity and lose all evidence of B-cell phenotype and instead acquire the phenotype of a histiocytic neoplasm. Little is known about the underlying genetic alterations that occur during this unusual process. Comparative genetic analysis of pre- and post-transformation/transdifferentiation would be one tool by which we could better understand how this phenomenon occurs.
\end{abstract}

Case presentation: Here we report the clinical, immunophenotypic and genetic features of a rare case of secondary malignant histiocytosis, Langerhans cell-type (Langerhans cell sarcoma) arising from a previous low grade follicular lymphoma. FISH analysis confirmed the presence of IgH/BCL2 rearrangement in both the low grade follicular lymphoma (FL) and transformed Langerhans cells sarcoma (LCS) samples, demonstrating a clonal relationship. Comparative whole exome sequencing was then performed, which identified a KRAS p. G13D mutation in the LCS that was not present in the FL.

Conclusions: This report highlights genetic alterations, in particular an acquired somatic KRAS mutation, that may occur during transdifferentiation, with additional significance of KRAS mutation as a possible therapeutic target in cases which otherwise would have limited treatment options.

Keywords: KRAS, Transdifferentiation, Langerhans cell sarcoma, Follicular lymphoma

\section{Background}

Follicular lymphoma (FL) is an indolent B-cell lymphoma composed of follicle center B cells [1]. While generally regarded as a manageable disease with a long survival rate, it can also progress to more aggressive forms of lymphoma, such as diffuse large B-cell lymphoma. Another rare and distinct form of disease progression is transdifferentiation from a B cell neoplasm to a neoplasm of another cell lineage [2-9]. In these instances, the neoplastic cells demonstrate lineage

\footnotetext{
* Correspondence: j-gao@northwestern.edu

${ }^{1}$ Department of Pathology, Northwestern University Feinberg School of

Medicine, 251 E Huron Street, Chicago, IL 60611, USA

Full list of author information is available at the end of the article
}

plasticity, manifested as an apparent shift in cellular phenotype from one lineage to another while still carrying shared underlying clonal genetic abnormalities. Most reported cases have involved transdifferentiation of chronic lymphocytic leukemia to histiocytic neoplasms $[2-4,6,8,9]$. Transdifferentiation is an elusive process, and the exact pathogenic mechanisms remain unknown. Comprehensive genetic analysis could provide a useful tool by which to identify genetic alterations that occur during transdifferentiation and may provide clues to the underlying pathogenesis of this process. Furthermore, as this form of transformation heralds a very poor prognosis, identification of targetable genetic mutations could offer therapeutic options in cases where there are few

(c) The Author(s). 2018 Open Access This article is distributed under the terms of the Creative Commons Attribution 4.0 International License (http://creativecommons.org/licenses/by/4.0/), which permits unrestricted use, distribution, and 
alternatives. Here, we described a rare occurrence of a low grade FL transdifferentiating into a Langerhans cell sarcoma (LCS) with acquisition of KRAS mutation. As comparative whole exome sequencing of pre- and post-transdifferentiation lymphoma samples has not yet been reported in the literature, this report will contribute to the presently limited understanding of this rare phenomenon.

\section{Case presentation}

Our patient had a long history of follicular lymphoma which initially presented as a localized neck mass. A biopsy of the mass showed low grade follicular lymphoma. The patient was treated with radiation therapy, but 3 years later, was diagnosed with diffuse large B cell lymphoma involving lung with discordant low grade follicular lymphoma in the marrow. After eight cycles of R-CHOP therapy (rituximab, cyclophosphamide, doxorubicin, vincristine, and prednisone), the patient went into remission. Unfortunately, 6 years later, the patient presented with skin and breast lesions as well as increased adenopathy, and was treated with rituximab, with some improvement of adenopathy. The skin nodules on the left arm were noted enlarging, a subsequent biopsy revealed low-grade follicular lymphoma, which responded to rituximab and bendamustine therapy. A few months later, the patient noted an enlarged inguinal lymph node, a biopsy of which showed Langerhans cell sarcoma. Despite 4 cycles of ICE chemotherapy and 8 weeks of ibrutinib, a PET/CT showed evidence of disease progression with diffuse intensely hypermetabolic soft tissue nodules and lymph nodes. Biopsy of one of the lymph nodes was consistent with diffuse large B cell lymphoma. The patient received palliative radiation therapy and passed away in a hospice facility. The patient's clinical history and treatment was summarized in Table 1.

\section{Histology}

The initial biopsy of the mass at presentation showed complete architectural effacement by a nodular lymphoid proliferation of small lymphocytes with elongated and cleaved nuclei (Fig. 1). Flow cytometric analysis identified a surface lambda light chain-restricted B-cell population that was CD10+ and CD5-. Diagnosis of a low grade follicular lymphoma was made.

Three years later, a transbronchial biopsy showed atypical mononuclear cell infiltrate composed of large cells with large nuclei and prominent nucleoli associated with frequent apoptotic bodies (Fig. 2). Immunohistochemical staining showed the large cells were positive for CD20 and $\mathrm{CD} 10$, and exhibited a moderate proliferation rate based on Ki-67 staining (40-50\%). These findings were consistent with diffuse large B-cell lymphoma. A concurrent bone marrow biopsy showed a paratrabecular lymphoid infiltrate composed of small centrocytes consistent with involvement by follicular lymphoma (Fig. 2). Flow cytometric analysis identified a monotypic, surface lambda light chain-restricted B-cell population that was dim CD10+ and CD5-, supporting involvement by follicular lymphoma.

The breast biopsy showed an abnormal lymphoid infiltrate composed predominantly of small lymphocytes with elongated nuclei and condensed chromatin. The abnormal lymphocytes were $\mathrm{CD} 20+, \mathrm{CD} 10+$, BCL-6+ and BCL-2+, consistent with recurrent follicular lymphoma (Fig. 3). A few months later, an enlarged inguinal lymph node showed effacement of architecture by an abnormal polymorphous infiltrate composed of many highly atypical large cells with admixed histiocytes, small lymphocytes, neutrophils,

Table 1 Summary of Clinical, Pathologic and Genetic Characteristics of Tumors

\begin{tabular}{|c|c|c|c|c|c|}
\hline Timeline & Location & Diagnosis & Immunophenotype of Neoplastic Cells & $\begin{array}{l}\text { Key FISH/Molecular } \\
\text { Characteristics }\end{array}$ & Therapy \\
\hline Year 1 & Neck mass & FL (Fig. 1) & $\begin{array}{l}\text { CD19+, CD10+, CD5-, surface lambda } \\
\text { restricted }\end{array}$ & NA & Radiation \\
\hline Year 3 & $\begin{array}{l}\text { Lung and bone } \\
\text { marrow }\end{array}$ & $\begin{array}{l}\text { Lung: DLBCL } \\
\text { Bone marrow: } \\
\text { FL (Fig. 2) }\end{array}$ & $\begin{array}{l}\text { Lung: CD20+, CD10+ } \\
\text { Bone marrow: CD19+, CD10+, CD5-, } \\
\text { surface lambda restricted }\end{array}$ & NA & $\mathrm{R}-\mathrm{CHOP}$ \\
\hline Year 9 & Skin and breast & FL (Fig. 3) & $\mathrm{CD} 20+, \mathrm{CD} 10+, \mathrm{BCL} 6+, \mathrm{BCL} 2+$ & $\begin{array}{l}\text { IgH/BCL2 fusion } \\
\text { KRAS wildtype } \\
\text { MYC extra copies; no MYC } \\
\text { rearrangement }\end{array}$ & R-Bendamustine \\
\hline Year 10 & $\begin{array}{l}\text { Inguinal lymph } \\
\text { node }\end{array}$ & LCS (Fig. 4) & $\begin{array}{l}\text { CD45+, CD43+, CD1a+, S100+, CD20-, PAX5-, } \\
\text { CD19-, CD79a-, CD10-, BCL6+ (focal) }\end{array}$ & $\begin{array}{l}\text { IgH/BCL2 fusion } \\
\text { KRAS p.G13D } \\
\text { MYC extra copies; } \\
\text { no MYC rearrangement }\end{array}$ & ICE, ibrutinib \\
\hline Year 10 & Ear mass & DLBCL & CD20+, CD10+, CD1a-, S100- & NA & $\begin{array}{l}\text { Palliative radiation } \\
\text { therapy }\end{array}$ \\
\hline
\end{tabular}




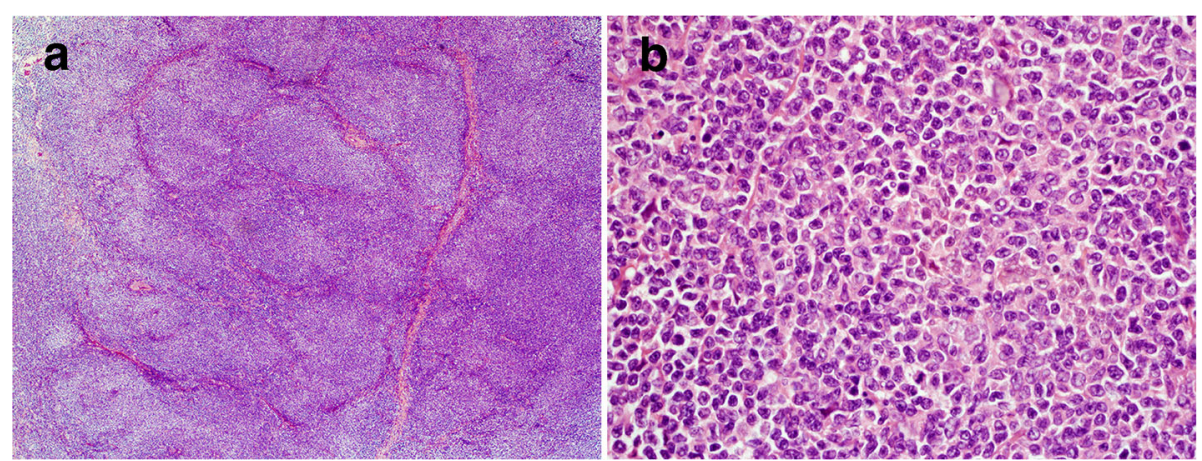

Fig. 1 Diagnostic low grade follicular lymphoma. Excisional biopsy reveals a nodular lymphoid proliferation of small lymphocytes with elongated nuclei (a H\&E, 100X; b H\&E, 600X). Flow cytometric analysis identifies a surface lambda light chain-restricted B-cell population that is CD10+ and CD5-

eosinophils and rare plasma cells (Fig. 4). The large pleomorphic cells had deeply convoluted nuclei and abundant cytoplasm. Immunohistochemical stains showed the large cells were positive for CD45, CD43, CD1a, and S-100. CD20 and PAX5 immunostains showed virtually no $\mathrm{B}$ cells present in the lesion. Flow cytometric analysis did not identify a distinct CD19+ B-cell population. The findings were consistent with Langerhans cell sarcoma.

\section{FISH analysis}

To establish the genetic relationship between the LCS and the patient's prior FL, FISH analysis for $t(14 ; 18)$ $B C L 2 / I g H$ was performed on the touch imprints of the recurrent breast follicular lymphoma and LCS. FISH analysis revealed the presence of $\mathrm{t}(14 ; 18) B C L 2 / \mathrm{IgH}$ fusion signals in $62 \%$ and $21 \%$ of the 200 cells analyzed in follicular lymphoma and LCS respectively (Fig. 3 and Fig. 5). In LCS, the fusion signal is only present within

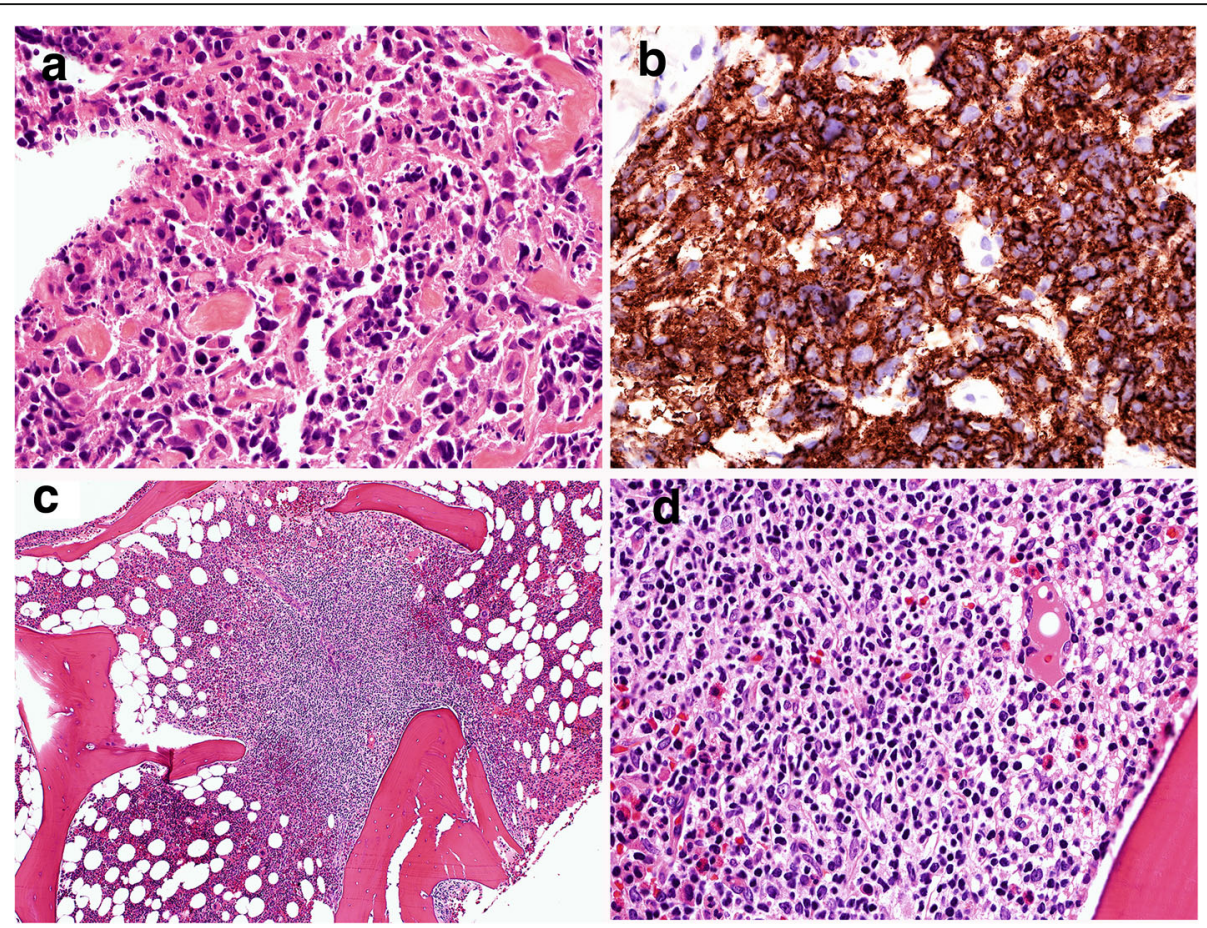

Fig. 2 Diffuse large B-cell lymphoma. A transbronchial biopsy of the lung mass shows a diffuse proliferation of CD20+ large cells with large nuclei, open chromatic, occasional nucleoli associated with frequent apoptotic bodies (a H\&E, 600X; b CD20 IHC, 600X). A bone marrow core biopsy shows an abnormal paratrabecular lymphoid infiltrate comprising of small lymphocytes with elongated nuclei (c H\&E, 200X; d H\&E, 600X). Flow cytometric analysis of the bone marrow aspirate (not shown) identified a monotypic, surface lambda light chain-restricted B-cell population that was dim CD10+ and CD5-, consistent with bone marrow involvement by follicular lymphoma 


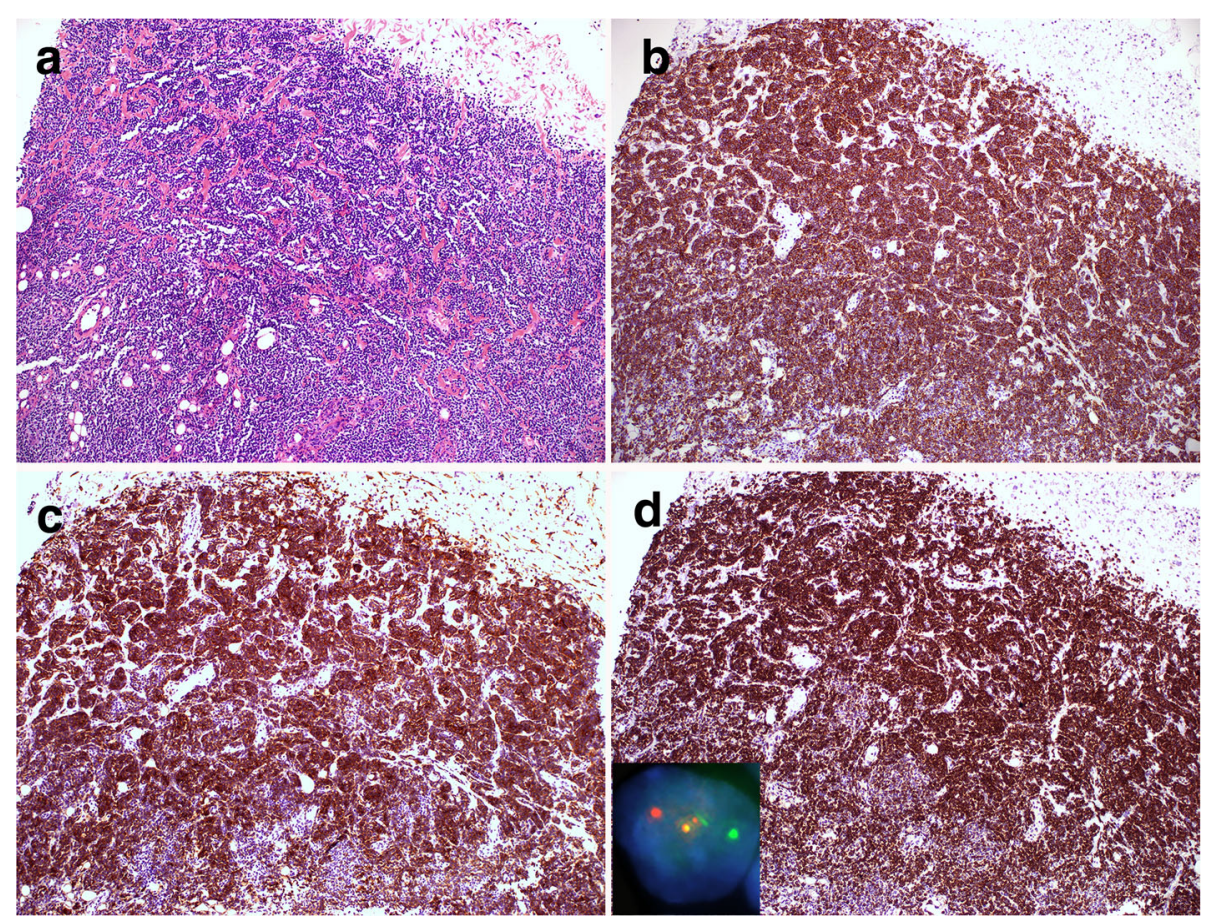

Fig. 3 Recurrent low grade follicular lymphoma. A needle core biopsy reveals an abnormal infiltrate of small lymphocytes that are predominantly CD20+ B cells with coexpression of CD10, BCL6 and BCL2, consistent with low grade follicular lymphoma (a H\&E, 200X, b CD20 IHC, $200 X ; \mathbf{c}$ CD10, 200X, d BCL2 IHC, 200X). Dual color dual fusion FISH analysis confirms the presence of IgH/BCL2 fusion signals resulting from $t(14 ; 18)$ in $62 \%$ of cells analyzed (Inset)

the sarcoma cells identified by their enlarged size and bizarre nuclear morphology, but not in any background inflammatory cells. And almost all the sarcoma cells harbor this translocation. Interestingly, the sarcoma cells displayed 4-5 copies of $I g H / B C L 2$, indicating amplification of fusion signals from $\mathrm{t}(14: 18)$ (Fig. 5). Using $M Y C$ break apart probes, both FL and Langerhans cell sarcoma sample were negative for $M Y C$ rearrangement; however, extra $M Y C$ signals were seen in $21 \%$ and $22 \%$ of the 200 cells analyzed in FL and LCS respectively, and in latter only present in the sarcoma cells. Molecular analysis was performed on the bone marrow aspirate with involvement of follicular lymphoma. The result was positive for clonal IGH gene rearrangement with $334 \mathrm{bp}$, 273 bp and 133 bp clonal bands by FR1, FR2 and FR3 biomed primers. Molecular analysis was also performed on the LCS and was negative for clonal immunoglobulin heavy chain rearrangement.

\section{Molecular analysis and whole exome sequencing}

Additional molecular analysis for BRAF V600E mutation, which has been reported in $38-57 \%$ of Langerhans cell histiocytosis but not as of yet in any cases of Langerhans cell sarcoma $[1,10,11]$, and was negative for this mutation.
Comparative whole exome sequencing was performed on paraffin-embedded tissue sections from the breast low-grade follicular lymphoma sample and Langerhans cell sarcoma sample. The tumor burden was estimated at $90 \%$ and $20-30 \%$ with median depth of read of 140 and 92 for the follicular lymphoma and Langerhans cell sarcoma samples, respectively. After filtering, there were 12 variants including 10 nonsynonymous SNVs and two inframe mutations shared by both the FL and LCS. The majority of SNVs, which had a variant allele frequency (VAF) close to 0.5 in both samples or had alternative allele frequency above or close to 0.01 in the $1000 \mathrm{~g}$ or ExAC database, may represent germline polymorphisms (Table 2, Additional file 1: Table S1). The shared CREBBP mutation (NM_001079846:exon29:c.4920_ 4922del:p.1640_1641del) has a VAF lower than other shared variants (0.31 in FL and 0.23 in LCS), and may represent a shared somatically acquired driver mutation. CREBBP is a histone modifier that is frequently mutated in FL $[12,13]$.

Twelve variants were present in the FL but not in the LCS. After filtering, there were 4 remaining nonsynonymous SNVs, 1 stopgain, and 3 inframe deletions (Table 2, Additional file 2: Table S2). Included among these variants were $K M T 2 D$ p.R5097* and BCL2 p.S203 N, which have been previously identified in FL $[14,15]$. 


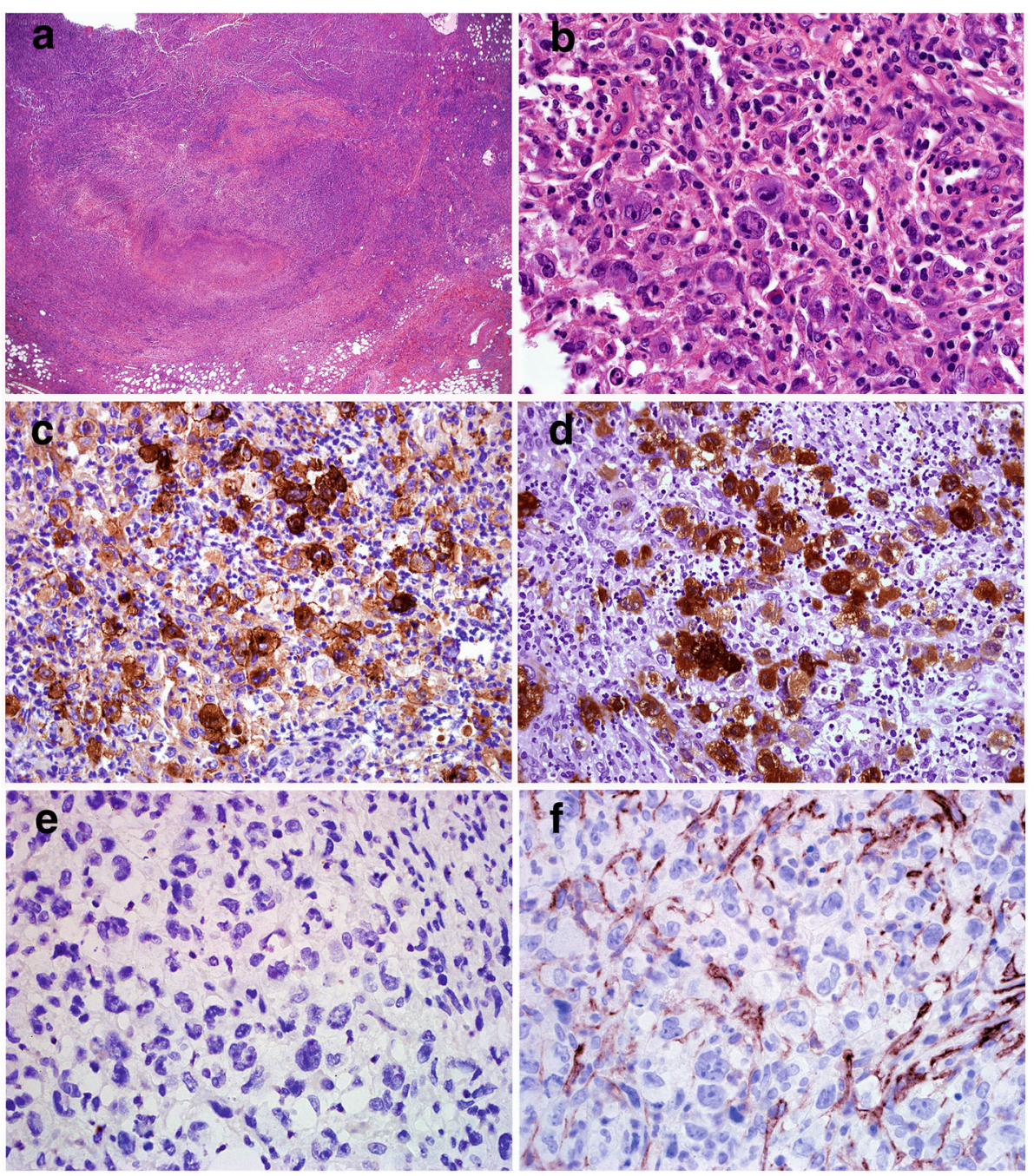

Fig. 4 Langerhans cell sarcoma. The inguinal lymph node biopsy shows a complete effacement of the lymph node architecture by an abnormal polymorphous infiltrate of histiocytes, small lymphocytes, neutrophils, eosinophils and rare plasma cells, with scattered and focal sheets of atypical large cells with convoluted nuclei (a H\&E, 100X, b H\&E, 600X). The large cells are CD1a + and S100+ (c CD1a IHC, 600X; d S100 IHC, 600X). The morphology and phenotype is consistent with a diagnosis of Langerhans cell sarcoma. The large cells were CD20-, CD19- (not shown), PAX5(not shown) and CD10- (e CD20 IHC, 600X; f CD10 IHC, 600X)

Although copy number alterations were not covered in this assay, analysis of allele frequency of commonly occurring SNVs can be a useful indicator of loss of heterozygosity (LOH). There were 14 nonsynonymous single nucleotide variants demonstrating potential $\mathrm{LOH}$ (i.e. heterozygous in the $\mathrm{FL}$ and homozygous in the LCS). None of the variants were considered deleterious (Table 2, Additional file 3: Table S3).

Thirty variants were identified in the LCS sample that were not present in the FL sample including 26 nonsynonymous SNVs, 2 frameshift insertions and 2 inframe deletions. After filtering out of benign or likely benign variants, the remaining variants included 11 nonsynonymous SNVs, 2 frameshift insertions and 2 inframe deletions (Table 2, Additional file 4: Table
S4). Most of the variants were of unknown significance, except KRAS p.G13D (Fig. 6), which is a well-known pathogenic variant in a variety of malignancies including carcinoma and hematopoietic neoplasms [16-18]. To confirm the presence of KRAS mutation, a real-time PCR analysis was also performed in both the FL and LCS. The results confirmed the presence of KRAS p.G13D in the LCS but not in the FL (data not shown).

\section{SNP microarray analysis}

Multiple chromosomal abnormalities were identified in the follicular lymphoma sample which include $6 p$ $\mathrm{CN}-\mathrm{LOH}, 6 \mathrm{q}$ deletion, small gain at 7p, 9p21.3 deletion (CDKN2A/2B genes included), small deletion at 10q, 


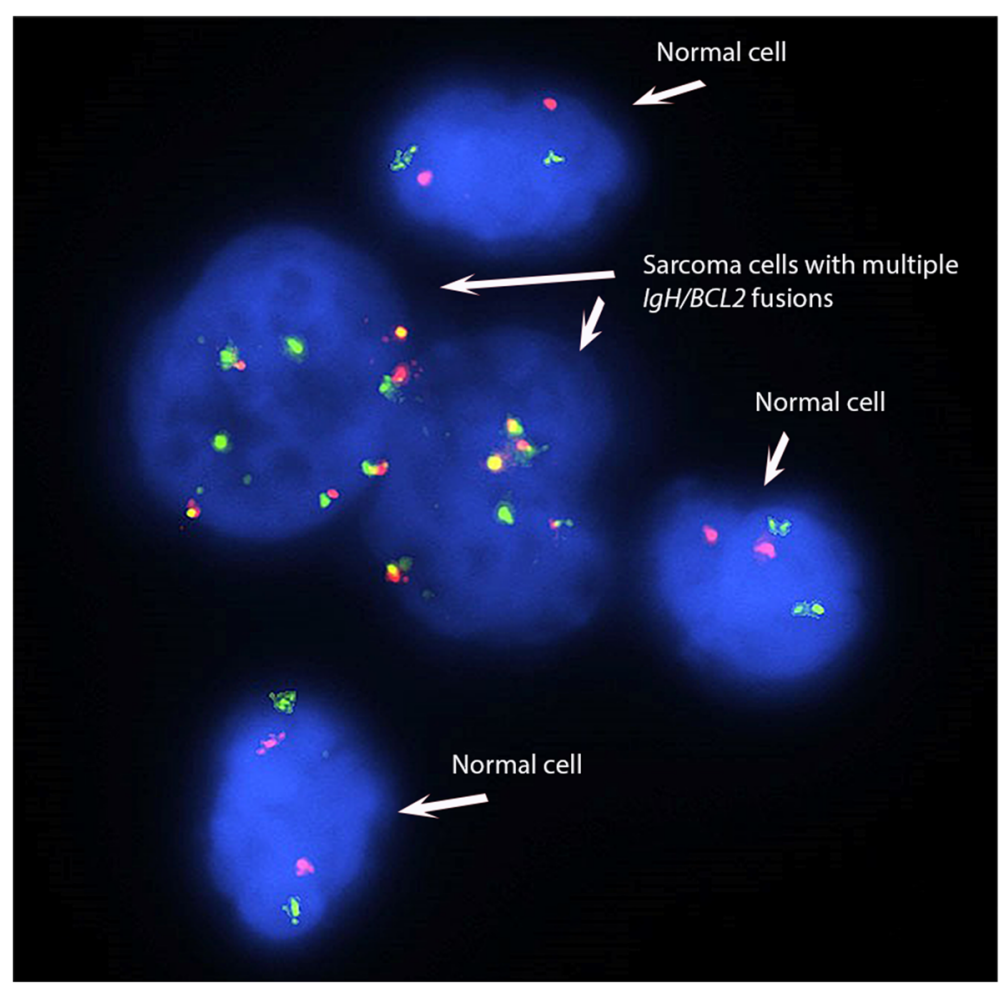

Fig. 5 Dual color dual fusion FISH analysis demonstrates multiple $\mathrm{g} H / B C L 2$ fusion signals resulting from $t(14 ; 18)$ in $21 \%$ of cells analyzed. The fusion signals are present only in the large sarcoma cells with but not in any of background inflammatory cells

small deletion at $12 \mathrm{q}$ (distal breakpoint within BCL7A gene), $16 \mathrm{p}$ deletion, small $16 \mathrm{p}$ gain, $2 \mathrm{CN}-\mathrm{LOHs}$ on $20 \mathrm{p}$ and some gains involving $20 \mathrm{p}$, a homozygous loss involving chromosome 22q11.22 involving the IGL gene and likely related to gene rearrangements in the tumor, a CNV gain involving chromosome 22q11.23 and gain of entire chromosome $\mathrm{X}$ (Table 3). However, there were no significant abnormalities identified in the Langerhans cell sarcoma sample with the exception of KRAS p. G13D and a CNV gain on chromosome 22q11.23, which was also observed in the follicular lymphoma sample (Table 3).

Table 2 Summary of Variants with Possible or Unknown Significance Present in Follicular Lymphoma or/and Langerhans Cell Sarcoma

\begin{tabular}{|c|c|c|}
\hline Variants Shared in FL and LCS & Variants present only in FL & Variants present only in LCS \\
\hline $\begin{array}{l}10 \text { nonsynonymous SNVs } \\
\text { SPEN p.K1064I } \\
\text { PDE4DIP p.R1867C } \\
\text { PDE4DIP p.E573V } \\
\text { PDE4DIP p.S438 L } \\
\text { PDE4DIP p.A127T } \\
\text { ARHGAP26 p.R293W } \\
\text { PMS2 p.I18V } \\
\text { ATM p.S49C } \\
\text { KMT2D p.P1131L } \\
\text { SPECC1 p.D688N }\end{array}$ & $\begin{array}{l}4 \text { nonsynonymous SNVs } \\
\text { IL2 p.V89 L } \\
\text { CDKN2A p.M1T } \\
\text { BCL2 p.S203 N } \\
\text { BCL2 p.A45T } \\
\text { SMARCA4 p.G883D }\end{array}$ & $\begin{array}{l}11 \text { nonsynonymous SNVs } \\
\text { BIN1 p.H283Y } \\
\text { TMEM200A p.G383R } \\
\text { DNAJB12 p.R13W } \\
\text { KRAS p.G13D } \\
\text { TRPV42 p.R249H } \\
\text { ADAD2 p.R299Q } \\
\text { KRTAP4-7 p.S113C } \\
\text { EMR2 p.C520F } \\
\text { ARMCX4 p.G1814E } \\
\text { ARMCX4 p.E1816G } \\
\text { ARMCX4 P.G1820E }\end{array}$ \\
\hline \multirow[t]{2}{*}{$\begin{array}{l}2 \text { inframe deletions } \\
\text { ZNF384 p.385_385del } \\
\text { CREBBP p.1640_1641del }\end{array}$} & $\begin{array}{l}1 \text { stopgain } \\
\text { KMT2D p.R5097X }\end{array}$ & $\begin{array}{l}2 \text { frameshift insertions } \\
\text { CAMTA1 p.N1271 fs } \\
\text { BCR p.G1049 fs }\end{array}$ \\
\hline & $\begin{array}{l}3 \text { inframe deletions } \\
\text { MLLT3 } \\
\text { MN1 p.524_524del } \\
\text { MN1 p.304_304del }\end{array}$ & $\begin{array}{l}2 \text { inframe deletions } \\
\text { ARID1A p.1333_1334del } \\
\text { NOTCH1 p.2411_2411del }\end{array}$ \\
\hline
\end{tabular}



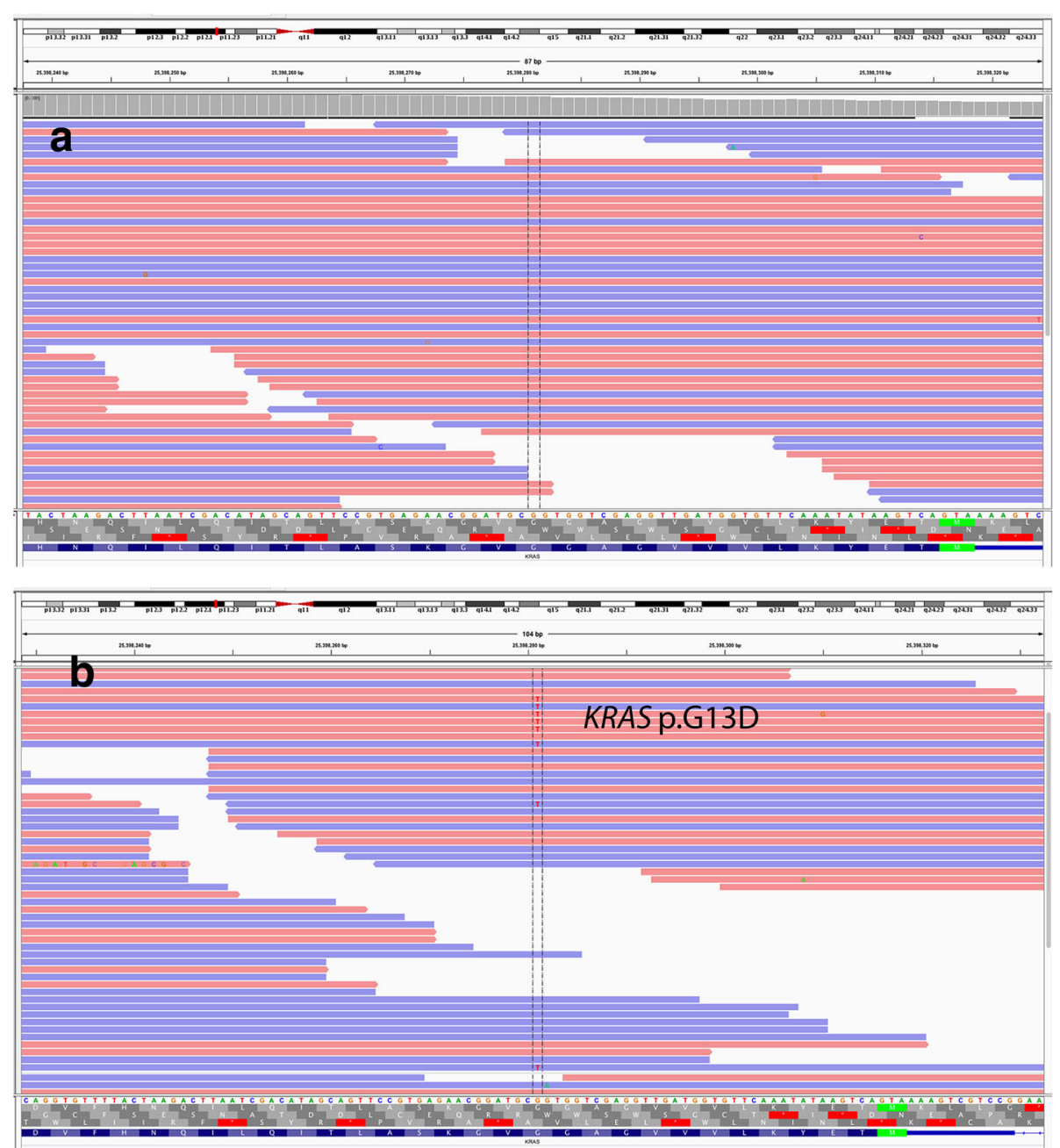

Fig. 6 Exome sequencing performed on the Langerhans cell sarcoma sample revealed a G13D mutation results in an amino acid substitution at position 13 in KRAS, from a glycine to an aspartic acid (Variant allele frequency: 0.24) (b). This KRAS p.G13D mutation was not present in the previous follicular lymphoma (a)

\section{Discussion and conclusions}

Classification of hematopoietic neoplasms is predicated on the assumption that the phenotype of a neoplastic cell is intrinsically linked to a predefined lineage and that this lineage determines the type and nature of the neoplasm. However, as demonstrated in cases of transdifferentiation, these associations are not hard and fast rules, as cells, both physiologic and neoplastic, can demonstrate lineage plasticity [3-9, 19-21]. Our patient's pathologic studies include a wide range of morphologic variation in the neoplastic clone, ranging from low grade FL to DLBCL to later on, LCS.

The mechanisms by which transdifferentiation occurs are largely unknown and previously described cases have relied upon molecular clonality or other genetic markers to establish a clonal relationship between what otherwise would appear as two phenotypically distinct neoplasms. Clonal IgH rearrangements have been reported in 39\% of sporadic cases of histiocytic/dendritic cell sarcomas [22] which may reflect the close relationship and possible common precursor between neoplasms of histiocytic differentiation and lymphoid differentiation $[4,6,7$, $9,19,23-25]$. In our case, while a clonal IgH rearrangement was detected in the FL from the fresh bone marrow sample, PCR analysis failed to identify a clonal $I g H$ rearrangement in LCS. The reason for the absence of clonal $I g H$ rearrangement in the LCS sample is not entirely clear, but could be due to additional genetic alterations affecting the specific binding of the PCR primers or decreased assay sensitivity in formalin fixed tissue. However, the clonal relationship between FL and LCS was definitively confirmed by FISH analysis, which able to readily identify the $\mathrm{t}(14 ; 18), \quad \operatorname{IgH} / B C L 2$ within morphologically-apparent sarcoma cells. The shared $\mathrm{t}(14 ; 18), I G H / B C L 2$ translocation in both the follicular lymphoma and LCS by FISH provided strong evidence 
Table 3 Chromosomal abnormalities in Follicular lymphoma and Langerhans cell sarcoma detected by SNP microarray

\begin{tabular}{|c|c|c|c|c|c|}
\hline$\overline{\text { Sample }}$ & Type & Chromosome & Cytoband & Size (Mb) & Comment \\
\hline \multirow[t]{14}{*}{ Follicular Lymphoma } & \multirow[t]{4}{*}{ LOSS } & 6 & q11.1-q27 & 109.02 & \\
\hline & & 9 & p21 & 0.74 & \multirow[t]{3}{*}{ CDKN2A gene } \\
\hline & & 10 & q24.1 & 1.43 & \\
\hline & & 16 & p13.3-p13.12 & 14.25 & \\
\hline & Homozygous loss & $22 q$ & q11.22 & 0.5 & \multirow[t]{5}{*}{ IGL gene } \\
\hline & \multirow[t]{5}{*}{ Gain } & 7 & p21.1-p15.3 & 4.18 & \\
\hline & & 16 & p11.2 & 1.01 & \\
\hline & & 20 & p12.2-p12.1 & 4.52 & \\
\hline & & 20 & p11.23-q13.33 & 44.31 & \\
\hline & & $x$ & p22.33-q28 & 155.27 & Whole chromosome gain \\
\hline & High copy gain & 22 & q11.23 & 0.05 & \multirow[t]{4}{*}{ CNV } \\
\hline & \multirow[t]{3}{*}{$\mathrm{CN}-\mathrm{LOH}$} & 6 & p25.3-p11.1 & 58.73 & \\
\hline & & 20 & p13-p12.2 & 9.45 & \\
\hline & & 20 & p12.1-p11.23 & 5.06 & \\
\hline \multirow[t]{2}{*}{ Langerhans cell sarcoma } & Point mutation & 12 & p12.1 & - & $\begin{array}{l}\text { p.G13D } \\
\text { c.38G > A }\end{array}$ \\
\hline & High copy gain & 22 & $q 11.23$ & 0.05 & CNV \\
\hline
\end{tabular}

of their clonal relationship, as this translocation is considered very specific for follicular lymphoma and has not been reported in any de novo sarcoma.

The shared CREBBP mutation (NM_001079846:exon29:c.4920_4922del:p.1640_1641del) is an interesting finding. $C R E B B P$ mutations, particularly mutations of the acetyltransferase domain, has been implicated as an early driver event in follicular lymphoma [26, 27]. Although the specific CREBBP mutation that we have detected in this case has not been previously reported in COSMIC database, it does occur within this functional acetyltransferase domain (p.1342-p.1649) [28, 29]. Thus, it could potentially represent a shared acquired somatic driver mutation and further support a model of direct transdifferentiation of LCS from antecedent follicular lymphoma rather than, for example, evolution from a common precursor neoplastic cell harboring an early translocation $\mathrm{t}(14 ; 18)$ event.

The additional SNP microarray results also suggest that the LCS likely originated from an earlier follicular lymphoma with $t(14 ; 18)$. As the LCS does not share the other complex genetic alterations with the exception of the shared CNV gain involving 22q11.23. However, it is worth pointing out that the LCS sample contains relatively low tumor burden (20-30\% by visual estimation) with a dense background of inflammatory cells, whereas the follicular lymphoma contains almost 100\% tumor cells. Therefore, other more subtle genetic alterations in the sarcoma cells may not be identified by the OncoScan SNP microarray which requires at least $25 \%$ tumor content.
The most intriguing finding in this case is the detection of KRAS p. G13D, a known pathogenic mutation, detected solely in the LCS sample. RAS mutations have been implicated in a number of hematologic malignancies, with NRAS mutations being more prevalent than KRAS mutations, particularly in myeloid neoplasms [30]. RAS-MAPK alterations have also been frequently described in hairy cell leukemia and multiple myeloma, but are more rarely seen in other lymphoproliferative disorders, such as diffuse large B-cell lymphoma [31, 32]. In studies analyzing mutational profiles of follicular lymphoma, a pathogenic role for RAS mutation in the development of follicular lymphoma has not been described [33, 34], although it has been reported as a rare event in cases of histologic transformation [35]. In a study of 55 primary DLBCL samples, KRAS p.G13D mutation was identified in 2 cases, thus is it possible that RAS mutations could drive the pathogenesis of a relatively rare subset of primary and secondary DLBCL [32]. A separate study described differential gene expression of genes in the MAPK signaling pathway, including NRAS, in cases of follicular lymphoma compared with transformed follicular lymphoma, suggesting activation of this pathway could be important in disease transformation [36]. In our case, the KRAS p.G13D may have arisen during the transformation process. Further studies to completely delineate clonal derivation were desired but precluded by the absence of material from original FL and subsequent DLBCL for additional testing. Nonetheless, the high prevalence of KRAS p.G13D 
mutation in the sarcoma cells, inferred from a variant allele frequency similar to the sarcoma tumor burden, suggests that it is a predominant mutation within the sarcoma cells.

Our finding raises a couple of important questions. What is the significance of KRAS mutation in histiocytic neoplasms? Could this mutation potentially drive transdifferentiation process itself? A recent abstract presentation [37] retrospectively reviewed cases of histiocytic sarcomas and discovered RAS/MAPK mutations in $44 \%$ $(n=8)$ of cases. KRAS p.G13D was present in 3 of the cases. Some of the cases of histiocytic sarcoma also possessed mutations that are common in B-cell lymphoma (such as MYD88, SOCS1, KMT2D, ARID1A) and furthermore, a subset of patients with histiocytic sarcoma had concurrent or prior history of B-cell lymphoma, including follicular lymphoma, which raised the possibility of transdifferentiation. This suggests that RAS/MAPK mutations are actually quite common in histiocytic neoplasms, even some that may be secondary to B-cell lymphoma, and that given their prevalence, may actively participate in driving this disease process.

In terms of Langerhans cell neoplasms, the RAS/ $M A P K$ signaling pathway has been strongly implicated as a pathogenic driver. Recent mouse models have demonstrated that expression of KRAS p.G12D mutation in lung myeloid cells induce the development of pulmonary Langerhans cell histiocytosis (LCH) [38]. NRAS p.Q61K mutations have also been reported at higher frequency in cases of pulmonary LCH [39], but have not thus far been seen in non-pulmonary cases. A study of 61 cases of non-pulmonary LCH reported BRAF V600E mutations in half of cases, also detected several other point mutations including TP53 p.R175H, KRAS p.G13D and $M E T$ p.E168D [10]. BRAF is an essential component of the RAS-RAF-MEK-ERK signaling cascade, which is triggered by binding of extracellular growth factor or cytokines to surface tyrosine kinase receptors, eventually leading to the modulation of downstream gene expression [40]. The activation of this pathway in LCH is further supported by the fact that MEK and ERK phosphorylation could be detected by immunohistochemistry in all cases of $\mathrm{LCH}$, regardless of $B R A F$ mutational status [41]. Activating mutations in BRAF other than V600E have also been detected in LCH [42-44]. Furthermore, cases without BRAF V600E mutation have demonstrated a high prevalence of somatic MAP2K1 mutation, which are mutually exclusive with $B R A F \mathrm{mu}-$ tations $[43,45]$. Thus, there is a substantial amount of evidence to suggest that the RAS-RAF-MEK-ERK signaling pathways is important to the evolution of $\mathrm{LCH}$.

LCS is an extremely rare malignancy thought to be derived from Langerhans histiocytes, and because of the scarcity of reported cases, the molecular pathogenesis of this entity is mostly unknown. However, it is not unreasonable to infer that some of the same pathways that drive $\mathrm{LCH}$ could also drive LCS. Interestingly, a recent report of histiocytic sarcoma transdifferentiation from B-lymphoblastic leukemia demonstrated NRAS p.G12D mutation, further suggesting RAS pathway alterations may be important in the transdifferentiation process [46]. It is also possible that other genetic or epigenetic changes which are as yet uncharacterized may contribute to lineage conversion and phenotypic switch seen during transdifferentiation.

Because of its prevalent role in neoplasms of multiple different organ systems, the RAS-RAF-MEK-ERK signaling pathway is also a target for novel therapeutics, including monoclonal antibodies and small molecule inhibitors, for example BRAF and MEK inhibitors in melanoma [47-49]. KRAS is one integral component of the RAS-RAF-MEK-ERK signaling pathway that acts upstream of BRAF [50]. Detection of KRAS mutation is now recommended in colorectal carcinoma, since its presence indicates resistance to targeted therapy against upstream signaling proteins (i.e. EGFR) [51]. Recent studies have shown specific efficacy of cetuximab in colorectal cancer patients with KRAS p.G13D mutations (the same mutation detected in our patient) and not other KRAS mutations [52, 53]. Ongoing studies in a variety of neoplasms examining RAS proteins and other downstream mediators, such as BRAF and MEK, as druggable targets offer additional therapeutic possibilities in patients with applicable genetic alterations, such as the current case [54-56].

It is important to recognize that while the results of whole exome sequencing in this case suggest complex genetic reprogramming may occur during the transdifferentiation process, this method may fail to identify all possible genetic abnormalities, including splice variants or chromosomal gains/losses. As such, additional genetic events may play a cooperative role in developing this aggressive disease. However, the detection of an activating KRAS mutation, which is not typically associated with B-cell lymphoma, combined with the growing body of evidence that this signaling pathway is essential to the development of Langerhans cell neoplasms, suggests that acquisition of this mutation could potentially drive or influence transdifferentiation.

In summary, this case describes transformation/transdifferentiation of a low grade FL into a LCS with detection of KRAS p.G13D mutation in the transformed sample. Our findings demonstrate characterize genetic events that may occur during transdifferentiation and further emphasizes the role of alterations of RAS-RAF-MEK-ERK signaling in the pathogenesis of Langerhans cell neoplasms. Importantly, the discovery of acquired KRAS mutation raises the possibility of targeted 
therapies (e.g. small molecule inhibitors) [50, 57] in the treatment of patients with these neoplasms who otherwise have limited therapeutic options.

\section{Materials and methods Pathology review}

H\&E sections from the patient's previous biopsies were retrieved from the Department of Pathology at Northwestern Memorial Hospital. The available archival materials were reviewed, along with associated ancillary studies, including immunohistochemical stains performed at the time of diagnosis, flow cytometric analysis and molecular analysis. Additional immunohistochemical stains, molecular and fluorescence in situ hybridization (FISH) analysis were performed in this study and are further described.

\section{Immunohistochemistry}

Immunohistochemical staining was performed on formalin-fixed, paraffin-embedded tissue. Antigen retrieval and immunohistochemical stain were performed on an automated immunostainer following the manufacturer's protocol (Ventana Medical Systems, Tuczon, AZ). The following predilute antibodies were used: CD3 (Ventana), BCL-2 (Cell Marque, Rocklin, CA), BCL-6 (Cell Marque), Ki-67 (Ventana), CD43 (Ventana), CD1a (Beckman Coulter, Miami, FL), S-100 (Ventana). The following antibodies were also used: CD20 (Dako, Carpinteria, CA), Pax-5 (1:10, Cell Marque), CD10 (1:30, Leica, Buffalo Grove, IL), CD45 (1:300, Dako). Positive and negative controls were performed with all cases and showed appropriate staining.

\section{Fluorescence in-situ hybridization (FISH)}

Interphase FISH with Vysis dual color break-apart MYC (8q24), and dual color break-apart BCL2 (18q21) probes (Abbott Molecular Inc., Des Plaines, IL) were performed on paraffin-embedded tissue sections. The $5^{\prime}$ and $3^{\prime}$ portions of the $M Y C$ and $B C L 2$ probes are labeled with SpectrumOrange and SpectrumGreen, respectively. Interphase FISH analysis with Vysis dual color dual fusion IGH (14q32) and BCL2 (18q21) probes were performed on the lymph node touch imprints. Negative and positive control slides were performed in parallel with the patient sample in the same hybridization process. A total of 100 interphase cells were evaluated for $M Y C$ and $B C L 2$ rearrangement independently by two laboratory technologists. An abnormal result was determined if the percentage of cells with abnormal FISH signals (i.e. separation of the $5^{\prime}$ and $3^{\prime} M Y C$ and $B C L 2$ probes due to a translocation or abnormal FISH signal patterns due to fusions) was above the relevant cut-off values at $95 \%$ confidence.
Immunoglobulin heavy chain (IGH) clonality assay

Molecular analyses were performed to assess for the presence of clonal IGH rearrangements. DNA was extracted from fresh bone marrow aspirate or formalin-fixed, paraffin-embedded tissue sections. DNA extraction and purification was performed on automated nucleic extraction instrument QIAsymphony SP using QIAsymphony DNA Mini Kit (Qiagen, Valencia, CA). Multiplex PCR-based clonality assays were performed following manufacturers' protocols (Invivoscribe, San Diego, CA, USA). The PCR products are detected by capillary gel electrophoresis on an ABI 3130XL genetic analyzer (Applied Biosystems, Foster City, CA, USA). An amplification band was considered clonal if the height of the peak was more than 3 times the height of the third highest peak in a given range of DNA sizes.

\section{BRAF V600E mutational analysis}

Analysis of BRAF V600E mutation was performed using COBAS 4800 BRAF Mutation assay according to the manufacturer's protocol (Roche Molecular Diagnostics). The results were reported as follows: i) V600E mutation detected, ii) V600E mutation not detected, or iii) invalid (ie, no result was obtained on the COBAS test). The COBAS BRAF Mutation assay can detect the BRAF V600E mutation at greater than $5 \%$ mutation level.

\section{Whole exome sequencing}

Whole exome sequencing was performed at the UCLA Technology Center for Genomics \& Bioinformatics. DNA was extracted from the formalin fixed paraffin embedded tissue sections from both the breast biopsy and inguinal lymph node. The library construction was performed using the SeqCap EZ System from NimbleGen (Roche NimbleGen, Inc. Madison, WI) according to the manufacturer's instructions. Briefly, genomic DNA was sheared, size selected to roughly 300 base pairs, and the ends were repaired and ligated to specific adapters and multiplexing indexes. Fragments were then incubated with SeqCap biotinylated DNA baits after LM-PCR and the hybrids were purified using streptavidin-coated magnetic beads. After amplification of 18 or less PCR cycles, the libraries were then sequenced on the HiSeq 3000 platform from Illumina, using 100-bp pair-ended reads.

The sequence data were aligned to the GRCh37 human reference genome using BWA v0.7.7-r411. PCR duplicates were marked using MarkDuplicates program in Picard-tools-1.115 tool set. GATK v3.2-2 was used for INDEL (insertions and deletions) realignment and base quality recalibration. Exome coverage was calculated using the bedtools. Samtools was used to call the SNVs (single nucleotide variants) and small INDELs. Varscan2 was used to call the somatic SNVs. All variants were 
annotated using the Annovar program. Copy number alterations and structural variants were not covered in this assay. The variants were ranked by SIFT and PolyPhen scores. A variant with SIFT score $<0.05$ is predicted as deleterious $(\mathrm{D})$, otherwise is tolerated $(\mathrm{T})$. A variant with Polyphen score $>0.9$ is predicted probably damaging, < 0.45 is predicted benign, between 0.45 and 0.9 is predicted possibly damaging. Variants were filtered out based on allele frequency of $>1 \%$ reported in 1000Genome, exAC (The Exome Aggregation Consortium); minimum read-depth of 30; any variants that were in the UTR or upstream/downstream of the gene; variants with a synonymous coding effect and variants within intronic region, unless they result in splice site mutations. Variants of $100 \%$ allele frequency in both tissues were considered germline and not included.

\section{KRAS mutational analysis}

Evaluation of KRAS mutational status was performed using the KRAS Mutation real-time PCR Analysis Kit (EntroGen, Tarzana, CA, USA) following the manufacturer's protocol. This assay employs allele-specific primers that are complementary to mutant variants of the KRAS genes. Detection of the amplification products was performed with the use of fluorescent hydrolysis probes. Probes tagged with the FAM fluorophore were complementary to the targets of KRAS genes. This assay identifies the following mutations in codon 12: G12S, G12D, G12V, G12C, G12A, G12R; in codon 13: G13D; and in codon 61: Q61H (61CAA > CAT), Q61L, Q61R, Q61H (61CAA > CAC).

\section{Single nucleotide polymorphism (SNP) microarray analysis}

SNP microarray analysis was performed using the OncoScan FFPE Express 3.0 assay kit (Affymetrix, Santa Clara, CA) as per manufacturer's recommendation. The assay utilizes Molecular Inversion Probe (MIP) technology, which is optimized for highly degraded FFPE samples (probe interrogation site of just 40 base pairs) (1-3). The assay employs 220,000 SNP MIP probes which ensure a resolution of 50-100 kb in selected genomic regions covering 900 cancer genes and of $300 \mathrm{~kb}$ outside of these regions. Briefly, 10 unstained FFPE tissue sections cut at $10 \mu \mathrm{M}$ were obtained and tumor was macrodissected using a hematoxylin and eosin -stained slide as a guide. DNA was extracted and purified from the samples using the QIAmp DNA FFPE Tissue Kit (Qiagen, Dusseldorf, Germany) according to the manufacturer's protocols. Extracted DNA was quantified using the Quant-iT PicoGreen dsDNA Assay Kit (Invitrogen, Carlsbad, CA, USA) following the manufacturer's protocol. A total of $80 \mathrm{ng}$ of DNA from each sample was annealed to the MIP probe panel. Annealed MIPs were circularized, followed by enzymatic removal of any un-ligated probe and template DNA. Remaining MIPs were linearized, amplified, enzymatically fragmented, and hybridized to oligonucleotide microarrays. The arrays were washed, scanned, and the results were analyzed and interpreted using OncoScan Console and Nexus Express for OncoScan 3 software (BioDiscovery, El Segundo, CA, USA) as previously described [58-60].

\section{Additional files}

Additional file 1: Table S1. Variants identified in both follicular lymphoma and Langerhans cell sarcoma. (DOCX $16 \mathrm{~kb}$ )

Additional file 2: Table S2. Variants identified in follicular lymphoma. (DOCX $16 \mathrm{~kb}$ )

Additional file 3: Table S3. Variants demonstrate a loss of heterozygosity during transformation from follicular lymphoma to Langerhans cell sarcoma. (DOCX 16 kb)

Additional file 4: Table S4. Variants identified in Langerhans cell sarcoma. (DOCX 17 kb)

\section{Availability of data and materials}

The datasets generated and/or analyzed during the current study are not publicly available but are available from the corresponding author on reasonable request.

\section{Authors' contributions}

SC, AA, MW, and JG collected the data, created the figures, and wrote the manuscript. $A B$ and $Y C$ provided critical review. $X L$ and $Y Z$ performed the FISH analysis. DD and JC performed molecular studies and analysis. AA and MW performed the SNP microarray studies and analysis. All authors read and approved the final manuscript.

Ethics approval and consent to participate

Exemption was received from the Northwestern University Institutional Review Board.

Consent for publication

Not applicable.

\section{Competing interests}

The authors declare that they have no competing interests.

\section{Publisher's Note}

Springer Nature remains neutral with regard to jurisdictional claims in published maps and institutional affiliations.

\section{Author details}

${ }^{1}$ Department of Pathology, Northwestern University Feinberg School of Medicine, 251 E Huron Street, Chicago, IL 60611, USA. ${ }^{2}$ Current address: Department of Pathology, University of Michigan, 5242 Medical Science Building 1, 1301 Catherine Street, Ann Arbor, MI 48109, USA. ${ }^{3}$ Current address: Department of Pathology, Memorial Sloan Kettering Cancer Center, 1275 York Ave, New York, NY 10065, USA. 'Diagnostic Molecular Biology Laboratory, Northwestern Memorial Hospital, 251 E Huron Street, Chicago, IL 60611, USA.

Received: 16 June 2018 Accepted: 2 October 2018

Published online: 15 October 2018

\section{References}

1. Swerdlow SHCE, Harris NL, Jaffe ES, Pileri SA, Stein H, Thiele J, Vardiman JW. WHO classification of Tumours of Haematopoietic and lymphoid tissues. In: WHO publications center; 2008. 
2. Ambrosio MR, De Falco G, Rocca BJ, et al. Langerhans cell sarcoma following marginal zone lymphoma: expanding the knowledge on mature B cell plasticity. Virchows Arch. 2015;467(4):471-80.

3. Chen W, Jaffe $R$, Zhang $L$, et al. Langerhans cell sarcoma arising from chronic lymphocytic lymphoma/small lymphocytic leukemia: lineage analysis and BRAF V600E mutation study. N Am J Med Sci. 2013;5(6): 386-91.

4. Feldman AL, Arber DA, Pittaluga S, et al. Clonally related follicular lymphomas and histiocytic/dendritic cell sarcomas: evidence for transdifferentiation of the follicular lymphoma clone. Blood. 2008;111(12): 5433-9.

5. Fraser CR, Wang W, Gomez M, et al. Transformation of chronic lymphocytic leukemia/small lymphocytic lymphoma to interdigitating dendritic cell sarcoma: evidence for transdifferentiation of the lymphoma clone. Am J Clin Pathol. 2009;132(6):928-39.

6. Shao H, Xi L, Raffeld M, et al. Clonally related histiocytic/dendritic cell sarcoma and chronic lymphocytic leukemia/small lymphocytic lymphoma: a study of seven cases. Mod Pathol. 2011;24(11):1421-32.

7. Wang $E$, Hutchinson $C B$, Huang $Q$, et al. Histiocytic sarcoma arising in indolent small B-cell lymphoma: report of two cases with molecular/genetic evidence suggestive of a 'transdifferentiation' during the clonal evolution. Leuk Lymphoma. 2010;51(5):802-12.

8. Wang E, Papalas J, Hutchinson CB, et al. Sequential development of histiocytic sarcoma and diffuse large b-cell lymphoma in a patient with a remote history of follicular lymphoma with genotypic evidence of a clonal relationship: a divergent (bilineal) neoplastic transformation of an indolent B-cell lymphoma in a single individual. Am J Surg Pathol. 2011;35(3):457-63.

9. West DS, Dogan A, Quint PS, et al. Clonally related follicular lymphomas and Langerhans cell neoplasms: expanding the spectrum of transdifferentiation. Am J Surg Pathol. 2013;37(7):978-86.

10. Badalian-Very G, Vergilio JA, Degar BA, et al. Recurrent BRAF mutations in Langerhans cell histiocytosis. Blood. 2010;116(11):1919-23.

11. Sahm F, Capper D, Preusser M, et al. BRAFV600E mutant protein is expressed in cells of variable maturation in Langerhans cell histiocytosis. Blood. 2012;120(12):e28-34.

12. Green MR. Chromatin modifying gene mutations in follicular lymphoma. Blood. 2018;131(6):595-604.

13. Korfi K, Ali S, Heward JA, Fitzgibbon J. Follicular lymphoma, a B cell malignancy addicted to epigenetic mutations. Epigenetics. 2017;12(5):370-7.

14. Green MR, Gentles AJ, Nair RV, et al. Hierarchy in somatic mutations arising during genomic evolution and progression of follicular lymphoma. Blood. 2013;121(9):1604-11.

15. Pasqualucci $L$, Khiabanian $H$, Fangazio $M$, et al. Genetics of follicular Iymphoma transformation. Cell Rep. 2014;6(1):130-40.

16. Ward AF, Braun BS, Shannon KM. Targeting oncogenic Ras signaling in hematologic malignancies. Blood. 2012;120(17):3397-406.

17. Malumbres M, Barbacid M. RAS oncogenes: the first 30 years. Nat Rev Cancer. 2003;3(6):459-65

18. Schubbert S, Shannon K, Bollag G. Hyperactive Ras in developmental disorders and cancer. Nat Rev Cancer. 2007;7(4):295-308.

19. Anjuere F, del Hoyo GM, Martin P, Ardavin C. Langerhans cells develop from a lymphoid-committed precursor. Blood. 2000;96(5):1633-7.

20. Feldman AL, Berthold F, Arceci RJ, et al. Clonal relationship between precursor T-lymphoblastic leukaemia/lymphoma and Langerhans-cell histiocytosis. Lancet Oncol. 2005;6(6):435-7.

21. Schmitt-Graeff AH, Duerkop H, Vollmer-Kary B, et al. Clonal relationship between langerhans cell histiocytosis and myeloid sarcoma. Leukemia. 2012;26(7):1707-10.

22. Chen W, Lau SK, Fong D, et al. High frequency of clonal immunoglobulin receptor gene rearrangements in sporadic histiocytic/dendritic cell sarcomas. Am J Surg Pathol. 2009;33(6):863-73.

23. Hou YH, Srour EF, Ramsey H, Dahl R, Broxmeyer HE, Hromas R. Identification of a human B-cell/myeloid common progenitor by the absence of CXCR4. Blood. 2005;105(9):3488-92.

24. Magni M, Di Nicola M, Carlo-Stella C, et al. Identical rearrangement of immunoglobulin heavy chain gene in neoplastic Langerhans cells and Blymphocytes: evidence for a common precursor. Leuk Res. 2002;26(12): 1131-3.

25. A clinical evaluation of the International Lymphoma Study Group classification of non-Hodgkin's lymphoma. The non-Hodgkin's lymphoma classification project. Blood. 1997;89(11):3909-18.
26. Green MR, Kihira S, Liu CL, et al. Mutations in early follicular lymphoma progenitors are associated with suppressed antigen presentation. Proc Natl Acad Sci U S A. 2015;112(10):E1116-25.

27. Garcia-Ramirez I, Tadros S, Gonzalez-Herrero I, et al. Crebbp loss cooperates with $\mathrm{BCl} 2$ overexpression to promote lymphoma in mice. Blood. 2017; 129(19):2645-56.

28. Delvecchio M, Gaucher J, Aguilar-Gurrieri C, Ortega E, Panne D. Structure of the p300 catalytic core and implications for chromatin targeting and HAT regulation. Nat Struct Mol Biol. 2013;20(9):1040-6.

29. Yuan LW, Giordano A. Acetyltransferase machinery conserved in p300/CBPfamily proteins. Oncogene. 2002;21(14):2253-60.

30. Ward AF, Braun BS, Shannon KM. Targeting oncogenic Ras signaling in hematologic malignancies. Blood. 2012;120(17):3397-406.

31. Vaque JP, Martinez N, Batlle-Lopez A, et al. B-cell lymphoma mutations: improving diagnostics and enabling targeted therapies. Haematologica. 2014;99(2):222-31.

32. Lohr JG, Stojanov P, Lawrence MS, et al. Discovery and prioritization of somatic mutations in diffuse large B-cell lymphoma (DLBCL) by wholeexome sequencing. Proc Natl Acad Sci U S A. 2012;109(10):3879-84.

33. Kridel $R$, Chan FC, Mottok A, et al. Histological transformation and progression in follicular lymphoma: a clonal evolution study. PLoS Med. 2016;13(12):e1002197.

34. Okosun J, Bodor C, Wang J, et al. Integrated genomic analysis identifies recurrent mutations and evolution patterns driving the initiation and progression of follicular lymphoma. Nat Genet. 2014;46(2):176-81.

35. Clark HM, Yano T, Sander C, Jaffe ES, Raffeld M. Mutation of the ras genes is a rare genetic event in the histologic transformation of follicular lymphoma. Leukemia. 1996;10(5):844-7.

36. Elenitoba-Johnson KS, Jenson SD, Abbott RT, et al. Involvement of multiple signaling pathways in follicular lymphoma transformation: p38-mitogenactivated protein kinase as a target for therapy. Proc Natl Acad Sci U S A. 2003:100(12):7259-64.

37. Shanmugam V, Sholl L, Fletcher CD, Hornick JL. RAS/MAPK Pathway Activation Defines a Common Molecular Subtype of Histiocytic Sarcoma. United States and Canadian Academy of Pathologists. Vancouver BC, CA; 2018

38. Kamata T, Giblett S, Pritchard C. KRAS(G12D) expression in lung-resident myeloid cells promotes pulmonary LCH-like neoplasm sensitive to statin treatment. Blood. 2017;130(4):514-26.

39. Mourah S, How-Kit A, Meignin V, et al. Recurrent NRAS mutations in pulmonary Langerhans cell histiocytosis. Eur Respir J. 2016;47(6):1785-96.

40. Montagut C, Settleman J. Targeting the RAF-MEK-ERK pathway in cancer therapy. Cancer Lett. 2009;283(2):125-34.

41. Badalian-Very G, Vergilio JA, Degar BA, Rodriguez-Galindo C, Rollins BJ. Recent advances in the understanding of Langerhans cell histiocytosis. $\mathrm{Br}$ J Haematol. 2012;156(2):163-72.

42. Zarnegar $\mathrm{S}$, Durham BH, Khattar $\mathrm{P}$, et al. Novel activating BRAF fusion identifies a recurrent alternative mechanism for ERK activation in pediatric Langerhans cell histiocytosis. Pediatr Blood Cancer. 2018;65(1).

43. Brown NA, Furtado LV, Betz BL, et al. High prevalence of somatic MAP2K1 mutations in BRAF V600E-negative Langerhans cell histiocytosis. Blood. 2014:124(10):1655-8.

44. Heritier S, Helias-Rodzewicz Z, Chakraborty R, et al. New somatic BRAF splicing mutation in Langerhans cell histiocytosis. Mol Cancer. 2017; 16(1):115.

45. Chakraborty R, Hampton OA, Shen $X$, et al. Mutually exclusive recurrent somatic mutations in MAP2K1 and BRAF support a central role for ERK activation in LCH pathogenesis. Blood. 2014;124(19):3007-15.

46. Thakral B, Khoury JD. Histiocytic sarcoma: secondary neoplasm or "transdifferentiation" in the setting of B-acute lymphoblastic leukemia. Blood. 2016;128(20):2475.

47. Hauschild A, Grob JJ, Demidov LV, et al. Dabrafenib in BRAF-mutated metastatic melanoma: a multicentre, open-label, phase 3 randomised controlled trial. Lancet. 2012;380(9839):358-65.

48. Chapman PB, Robert C, Larkin J, et al. Vemurafenib in patients with BRAFV600 mutation-positive metastatic melanoma: final overall survival results of the randomized BRIM-3 study. Ann Oncol. 2017;28(10):2581-7.

49. Flaherty KT, Robert $C$, Hersey $P$, et al. Improved survival with MEK inhibition in BRAF-mutated melanoma. N Engl J Med. 2012;367(2):107-14.

50. Morkel M, Riemer P, Bläker H, Sers C. Similar but different: distinct roles for KRAS and BRAF oncogenes in colorectal cancer development and therapy resistance. Oncotarget. 2015;6(25):20785-800. 
51. Misale $S$, Yaeger R, Hobor $S$, et al. Emergence of KRAS mutations and acquired resistance to anti-EGFR therapy in colorectal cancer. Nature. 2012; 486(7404):532-6.

52. De Roock W, Jonker DJ, Di Nicolantonio F, et al. Association of kras p.g13d mutation with outcome in patients with chemotherapy-refractory metastatic colorectal cancer treated with cetuximab. JAMA. 2010;304(16): $1812-20$.

53. Gajate P, Sastre J, Bando I, et al. Influence of KRAS p.G13D mutation in patients with metastatic colorectal Cancer treated with Cetuximab. Clin Colorectal Cancer. 2012;11(4):291-6.

54. Cox AD, Fesik SW, Kimmelman AC, Luo J, Der CJ. Drugging the undruggable RAS: Mission possible? Nat Rev Drug Discov. 2014;13(11) 828-51.

55. Kim J, McMillan E, Kim HS, et al. XPO1-dependent nuclear export is a druggable vulnerability in KRAS-mutant lung cancer. Nature. 2016;538(7623): 114-7.

56. Abla O, Weitzman S. Treatment of Langerhans cell histiocytosis: role of BRAF/MAPK inhibition. Hematology Am Soc Hematol Educ Program. 2015; 2015:565-70.

57. Ostrem JML, Shokat KM. Direct small-molecule inhibitors of KRAS: from structural insights to mechanism-based design. Nat Rev Drug Discov. 2016; 15(11):771-85.

58. Foster JM, Oumie A, Togneri FS, et al. Cross-laboratory validation of the OncoScan(R) FFPE assay, a multiplex tool for whole genome tumour profiling. BMC Med Genet. 2015;8:5.

59. Wang Y, Cottman M, Schiffman JD. Molecular inversion probes: a novel microarray technology and its application in cancer research. Cancer Genet. 2012;205(7-8):341-55.

60. Carter MD, Gaston D, Huang W-Y, et al. Genetic profiles of different subsets of Merkel cell carcinoma show links between combined and pure MCPyVnegative tumors. Hum Pathol. 2018;71:117-25.

Ready to submit your research? Choose BMC and benefit from:

- fast, convenient online submission

- thorough peer review by experienced researchers in your field

- rapid publication on acceptance

- support for research data, including large and complex data types

- gold Open Access which fosters wider collaboration and increased citations

- maximum visibility for your research: over $100 \mathrm{M}$ website views per year

At $\mathrm{BMC}$, research is always in progress.

Learn more biomedcentral.com/submissions 